\title{
Isolation of Extracellular Cobalt-free Corrinoid from Methanosarcina barkeri
}

\author{
Satoshi FuKUZaKI, Naomichi Nishio and Shiro NAGAI* \\ Department of Fermentation Technology, Faculty of Engineering, \\ Hiroshima University, Saijo-cho, Higashi-Hiroshima 724, Japan
}

Received March 27, 1989

\begin{abstract}
Cobalt-free corrinoids (CFCs) were isolated from Methanosarcina barkeri Fusaro cells growing on a methanol minimum medium. The methanogen cells excreted a trace of CFCs $(9.1 \mu \mathrm{g} / \mathrm{l})$ into the culture medium when cobalt-deficient methanol medium was used. Several CFCs were separated by column chromatographies on ion exchangers and paper electrophoresis, where a major CFC showed a similar characteristic to that of nucleotide-free corrinoid, Factor $B$ (cobinamide), suggesting to be hydrogenobinamide. By chemical insertion of $\mathrm{Co}^{2+}, \mathrm{Cu}^{2+}$, and $\mathrm{Zn}^{2+}$ into $\mathrm{CFCs}$, the corresponding corrinoid and its metal analogues were observed. Bioassay using Escherichia coli 215 revealed that the major CFC (a yellow product obtained after alkaline treatment) and its copper and zinc analogues were inactive as cobalamin but were active as antimetabolites of cobalamin. However, the CFC greatly stimulated the cell growth of $M$. barkeri grown under cobalt-deficient conditions.
\end{abstract}

It has been reported that cobalt-free corrinoids (CFCs) which contain no cobalt in the corrin skeleton had been isolated from certain species of photosynthetic bacteria ${ }^{1 \sim 4)}$ and Streptomyces olivaceus ${ }^{5}$ grown under cobaltdeficient conditions. However, CFCs have not so far been observed in other corrinoidproducing organisms. Several metal analogues of corrinoids have been prepared chemically from $\mathrm{CFCs}$ and their biological properties have also been investigated. ${ }^{2.3,6 ~ 9)}$ These researches showed that CFCs and their metal analogues were biologically inactive and acted as antimetabolites of cobalamin in vitamin B12-requiring auxotrophs. On the other hand, studies on human bone marrow cells ${ }^{10)}$ showed that neither of methyl- and 5'-deoxyadenosylrhodibalamin induce a vitamin B-12 deficient pattern in normoblastic bone marrow cells and methylrhodibalamin, like methylcobalamin, corrects the abnormality in vitamin B-12 deficient megaloblastic bone marrow cells but 5 deoxyadenosylrhodibalamin worsened their abnormality. These antimetabolite properties of CFC and metal analogues may be applic- able clinically.

Although methanogens are known to contain considerable amounts of corrinoids, ${ }^{11,12)}$ CFCs have not been observed. We previously reported production of extracellular corrinoids from methanol by Methanosarcina barkeri in a fixed-bed reactor. ${ }^{13)}$ In this system, when cobalt-deficient medium was supplied to the reactor, some unusual orange compounds were observed during the isolation and purification of corrinoids. These compounds clearly differed from porphyrins and showed an orange fluorescence under UV light suggesting that these compounds might be CFCs. In this paper, we describe the isolation of CFCs from the culture filtrate of $M$. barkeri and the effects of CFCs on the growth of E. coli 215, a vitamin B-12-requiring auxotroph, and $M$. barkeri.

\section{Materials and Methods}

Microorganism. Methanosarcina barkeri Fusaro (DSM 804), obtained from the Deutsche Sammlung von Microorganismen (Göttingen, Federal Republic of

* To whom correspondence should be addressed. 
Germany), was used throughout this study.

Cultivation. The basal minimum medium containing cobalt chloride was the same as described previously (methanol $=8 \mathrm{~g} / \mathrm{l}){ }^{13)}$ Fixed-bed culture was done in a cylindrical glass column reactor $(2.7 \mathrm{~cm}$ diameter $\times 17 \mathrm{~cm}$ length) with a working volume of $85 \mathrm{ml}$. The reactor was packed with diatomaceous clay (particle size $=4 \sim 8 \mathrm{~mm}$, diameter) as a support material by leaving about a $50 \%$ void fraction as described previously. ${ }^{137}$ A cobalt-deficient medium eliminating cobalt from the basal medium was started when a constant methanol consumption rate $(80 \mathrm{~g} / 1$-reactor $\cdot$ day) was achieved at a space velocity (SV) of $10.4 \mathrm{day}^{-1}$. During the continuous operation, the reactor was kept in dark condition.

Isolation of CFCs. CFCs were isolated from the culture effluent of $M$. barkeri as described by Dresow et al ${ }^{4\}}$ (see Fig. 1). The CFC fractions thus obtained were further purified and separated into 9 fractions (Fig. 1) by paper electrophoresis on Toyo filter paper No. 526 (Toyo Roshi Ltd., Japan) with I $\mathrm{N}$ acetate buffer ( $\mathrm{pH} \mathrm{2.5)}$ and $\mathrm{m} / 50$ potassium phosphate buffer ( $\mathrm{pH} 7.5$ ) containing $0.01 \%$ $\mathrm{KCN}$, and by ascending paper chromatography on the same filter paper with following solvents; water saturated 2-butanol-acetic acid-5\% KCN solution (100:1:0.2) and water saturated 2-butanol-5\% $\mathrm{KCN}$ solution $(100: 0.2) .^{13}$ The CFC yields in the fractions were estimated based on the molar extinction coefficients, ${ }^{14)} \lambda_{\max }: 524,498$, and $329 \mathrm{~nm}\left(\varepsilon: 20140,18640\right.$, and $48370 \mathrm{M}^{-1} \cdot \mathrm{cm}^{-1}$, respectively) and molecular weight of hydrogenobinamide (MW: 951), analogue of cobinamide (Factor B) as a tentative molecular weight.

Preparation of metal analogues. Chemical insertions of metals into a major CFC (CFC-1) were done by the methods of Toohey $^{2)}$ and Koppenhagen et al. ${ }^{6}$ : cobalt insertion, $0.8 \mathrm{ml}$ of $\mathrm{CFC}$ solution ( $4 \mathrm{nmol}$ ) was mixed with $0.1 \mathrm{ml}$ of $0.1 \mathrm{M} \mathrm{CoCl}_{2}$ solution and $0.1 \mathrm{ml}$ of $0.1 \% \mathrm{KCN}$ solution, and reacted at $20^{\circ} \mathrm{C}$ for $48 \mathrm{hr}(\mathrm{pH} 8.7)$; copper insertion, $0.8 \mathrm{ml}$ of CFC solution ( $4 \mathrm{nmol}$ ) was mixed with $0.1 \mathrm{ml}$ of $1 \% \mathrm{CuSO}_{4}$ solution and reacted at $100^{\circ} \mathrm{C}$ for $1 \mathrm{~min}$ or at $20^{\circ} \mathrm{C}$ for $16 \mathrm{hr}(\mathrm{pH} 2.6 \sim 6.0)$; zinc insertion, $0.8 \mathrm{ml}$ of $\mathrm{CFC}$ solution ( $4 \mathrm{nmol}$ ) was mixed with $0.1 \mathrm{ml}$ of 1 or $10 \% \mathrm{Zn}\left(\mathrm{CH}_{3} \mathrm{COOH}\right)_{2}$ solution and reacted at $20^{\circ} \mathrm{C}$ for $16 \mathrm{hr}$ (pH 6.8). All reactions were done in the dark.

Biological properties of CFCs. The biological activities of a CFC (CFC-1) and its metal analogues were measured using Escherichia coli 215, ${ }^{15)}$ a vitamin B-12-requiring auxotroph, as a test organism with or without cyanocobalamin. The effects of CFC-1 on the growth of $M$. barkeri were also tested in serum vials (about $125 \mathrm{ml}$ ) containing $50 \mathrm{ml}$ of medium with or without cobalt salt as follows: $M$. barkeri was subcultured twice in the cobalt-deficient medium and then inoculated into the three media, i.e., basal medium, cobalt-deficient medium and cobalt-deficient me- dium containing CFC (final concentration $=120 \mathrm{~nm}$ ). Cultures were then incubated at $37^{\circ} \mathrm{C}$ without shaking.

Analysis. Cell growth activity in the fixed-bed reactor was monitored by gas $\left(\mathrm{CH}_{4}+\mathrm{CO}_{2}\right)$ production and effluent methanol concentration. The composition of evolved gas was identified and methanol concentrations were measured by gas chromatography (TCD and FID, respectively). ${ }^{13)}$ The whole-cell protein of $M$. barkeri was measured as described previously. ${ }^{14)}$

Chemicals. Amberlite XAD-2 and the cellulose ion exchanger (type: DEAE 32 and CM23) were products of Rohm and Hass Co., U.S.A., and Serva Feinbiochemica GmbH and Co., U.S.A. Vitamin B-12 Factor III (5hydroxybenzimidazolyl cobamide, FIII), vitamin B-12 Factor B (cobinamide, FB) and vitamin B-12 Factor $\nabla_{1 \mathrm{a}}$ (cobyric acid, $F \nabla_{1 \mathrm{a}}$ ) were purified from digestion sludges. ${ }^{16)}$ Cyanocobalamin (5,6-dimethylbenzimidazoly] cobamide, CN-B-12) was obtained from Pierrel, Italy. Diatomaceous clay was obtained from a commercial source.

\section{Results and Discussion}

\section{Isolation of $C F C$}

After establishing a steady state culture of M. barkeri cells (methanol consumption rate $=$ $80 \mathrm{~g} / \mathrm{l}$-reactor $\cdot$ day, extracellular corrinoid concentration $=7.5 \mathrm{mg} / 1$, and $\mathrm{SV}=10.4$ day $^{-1}$ ), the cobalt-deficient medium was put into the reactor. For 3 weeks of cultivation cobalt deficiency did not distinctly affect the rate of methanol consumption and gas production, while total corrinoid concentration in the effluent decreased sharply (data not shown), suggesting the possibility of CFC formation. Therefore, the CFC formed was isolated and purified by column chromatography on DEAE- and CM-cellulose, paper electrophoresis, and paper chromatography. Nine fractions of orange fluorescent compounds were detected under UV light (366 nm) (Fig. 1). Figure 2 shows a typical absorption spectrum of a major CFC (CFC-1). The absorption maxima $(524,497,329$, and $269 \mathrm{~nm}$, respectively) coincide with those of CFCs reported previously. ${ }^{1,2)}$ All the other compounds had similar absorption spectra with the same absorption maxima but different absorption ratios. These compounds were changed to yellow compounds (Y-CFCs) at alkaline $\mathrm{pH}(>8.0)$, 


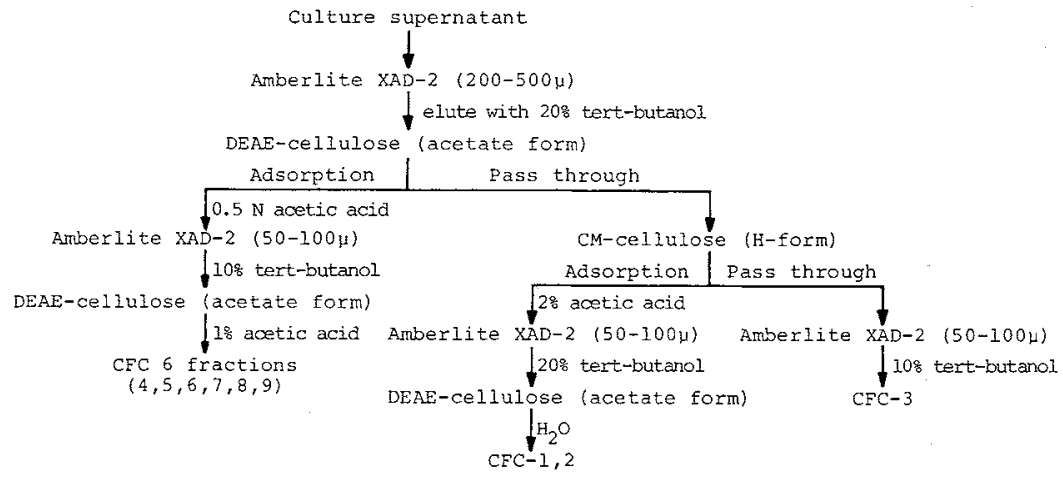

Fig. 1. Isolation of Cobalt-free Corrinoids from $M$. barkeri Fusaro.

$\mathrm{CFC}$ fractions were detected by orange-red fluorescence after paper electrophoresis and paper chromatography.

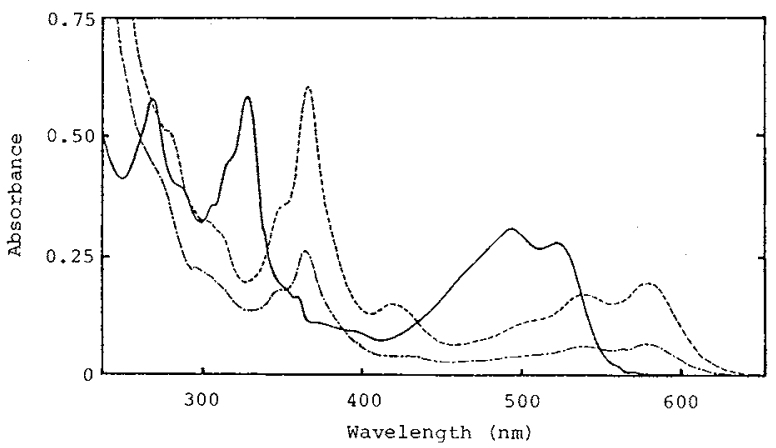

Fig. 2. Absorption Spectra of a Main Cobalt-free Corrinoid (CFC-1), Factor B, and a Cobalt Derivative of CFC-1 Produced by $M$. barkeri Fusaro.

- absorption spectrum of CFC-1 in water at $\mathrm{pH} 4.5$, (maxima: 524, 497, 329, and $269 \mathrm{~nm}$ ); ----, absorption spectrum of Factor B isolated from $M$. barkeri Fusaro in $0.1 \mathrm{M} \mathrm{KCN}$ aqueous solution at $\mathrm{pH} 10$, (maxima: 584, 543, and $369 \mathrm{~nm}$ ); — - absorption spectrum of the cobalt derivative of CFC- 1 in $0.1 \mathrm{M} \mathrm{KCN}$ aqueous solution at $\mathrm{pH} 8.0$, (maxima: 584,543 , and $369 \mathrm{~nm}$ ).

and this change was irreversible. When cobalt chloride was added to an alkaline solution of CFC-1 in the presence of $\mathrm{KCN}(0.01 \%)$, the absorption spectrum of the product in this reaction became identical with that of dicyanocorrinoid (FB) giving absorption maxima at 584, 543, and 369 (see Fig. 2). These facts suggest that these compounds isolated from culture filtrate of $M$. barkeri might be CFCs.

Figure 3 shows the behaviors of $9 \mathrm{CFC}$ fractions in paper electrophoresis compared with the authentic corrinoid compounds. Under acidic conditions, there were no fractions which corresponded to any of the two complete forms of corrinoid (CN-B-12 and
FIII) and their behaviors were similar to those of the base-free corrinoids ( $F B$ and $F \nabla_{1 a}$ ) which were the main corrinoids produced by $M$. barkeri in the presence of cobalt chloride. ${ }^{13)}$ On the other hand, under neutral conditions, the behaviors of CFC-1, 3 and 4, 5 were similar to those of $\mathrm{FB}$ and $\mathrm{F} V_{1 \mathrm{a}}$, respectively, suggesting the structural similarity of $\mathrm{CFC}-1$ and 3 to $\mathrm{FB}$ and of $\mathrm{CFC}-4$ and 5 to F $V_{1 \mathrm{a}}$. CFC-6 to 9 might have more carboxylic acid side chains on their corrin nucleus.

$R f$ values of CFC fractions on paper chromatography are shown in Table I. $R f$ values of CFC-1 closely resemble those of CFC-3 together with same electrophoretic behaviors 

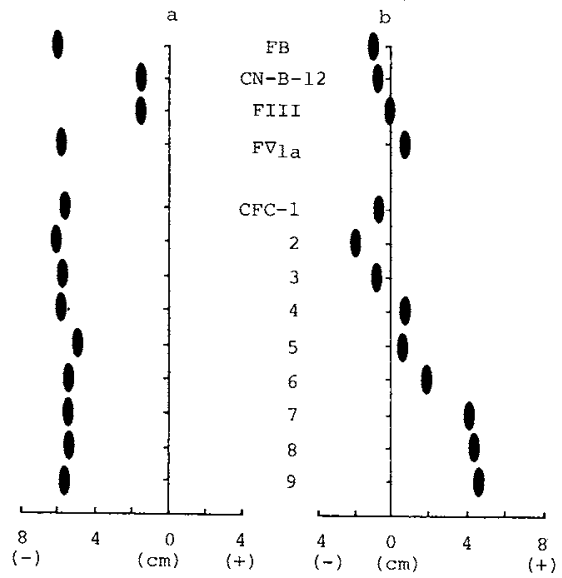

Fig. 3. Paper Electrophoretic Behavior of Cobalt-free Corrinoids Isolated from $M$. barkeri Fusaro.

a) acidic conditions: $14.4 \mathrm{~V} / \mathrm{cm}$ for $2 \mathrm{hr}$ in acetate buffer (pH 2.4) containing $0.01 \% \mathrm{KCN}$. b) neutral conditions: $8.9 \mathrm{~V} / \mathrm{cm}$ for $2 \mathrm{hr}$ in phosphate buffer $(\mathrm{pH} 7.5$ ) containing $0.01 \%$ KCN. FB, cobinamide; CN-B-12, cyanocobalamin (5,6-dimethylbenzimidazolyl cobamide); FIII, 5-hydroxybenzimidazolyl cobamide; $F \nabla_{\text {la }}$, cobyric acid.

mentioned above. These data could be used to distinguish between CFCs formed, indicating that $M$. barkeri could produce at least 8 kinds of CFCs in a cobalt-deficient medium.

The relative yields of CFCs isolated from the culture filtrate of $M$. barkeri are shown in Table II. Total amounts of CFCs were $9.1 \mu \mathrm{g} / 1$-effluent giving $94.2 \mu \mathrm{g} /$ l-reactor $\cdot$ day, where $54 \%$ of the CFCs were CFC-1. Although the CFC concentration is fairly low compared with that of photosynthetic bacteria $^{4)}$ and the chemical structures of these compounds are not fully known, this is the first cobalt-free corrinoid found in archaebacteria.

\section{Biological activity of $C F C$ for E. coli 215}

The biological activities of a major $\mathrm{CFC}$ (CFC-1), its yellow product ( $\mathrm{Y}-\mathrm{CFC}-1)$, and its copper and zinc analogues were examined using a vitamin B-12-requiring auxotroph, $E$. coli 215 . In the absence of cyanocobalamin, none of them showed significant growthpromoting activity (= vitamin B-12 activity) (data not shown), but in the presence of cyanocobalamin (14.8 pm), these compounds,
Table I. Paper Chromatography of Cobalt-free CORRINOIDS ISOLATED FROM $M$. barkeri FUSARO

\begin{tabular}{|c|c|c|}
\hline \multirow{2}{*}{$\begin{array}{l}\text { CFCs } \\
\text { isolated }\end{array}$} & \multicolumn{2}{|c|}{$R f$} \\
\hline & Solvent A & Solvent B \\
\hline 1 & 0.45 & 0.29 \\
\hline 2 & 0.48 & 0.50 \\
\hline 3 & 0.45 & 0.28 \\
\hline 4 & 0.43 & 0.08 \\
\hline 5 & 0.38 & 0.10 \\
\hline 6 & 0.46 & 0.06 \\
\hline 7 & 0.60 & 0.01 \\
\hline 8 & 0.24 & 0.04 \\
\hline 9 & 0.59 & 0.03 \\
\hline
\end{tabular}

Solvent A: water saturated 2-butanol-acetic acid-5\% $\mathrm{KCN}$ solution $(100: 1 ; 0.2)$.

Solvent B: water saturated 2-butanol-5\% $\mathrm{KCN}$ solution $(100: 0.2)$.

Table II. Relative Yields of CoBalt-FreE CORRINOIDS ISOLATED FROM M. barkeri FUSARO

\begin{tabular}{ccc}
\hline \multirow{2}{*}{$\begin{array}{c}\text { CFCs } \\
\text { isolated }\end{array}$} & \multicolumn{2}{c}{ Amount of CFC } \\
\cline { 2 - 3 } & (nmol/1-effuent) & $(\%)$ \\
\hline 1 & 5.15 & 54.1 \\
2 & 1.05 & 11.0 \\
3 & Trace & - \\
4 & 0.62 & 6.5 \\
5 & 0.45 & 4.7 \\
6 & 0.50 & 5.2 \\
7 & 1.21 & 12.7 \\
8 & 0.34 & 3.6 \\
9 & 0.22 & 2.3 \\
\hline
\end{tabular}

except the zinc analogue, acted as antimetabolites of vitamin B-12 (see Table III) as described by Koppenhagen. ${ }^{17)}$ Especially, CFC-1 and copper analogue were potent inhibitors. Their inhibition index values (approximately 0.5 ) were higher than (2 to 4 ) Friedrich's S-102, hydrogenobalamin, CFCs in Chromatium, cupribalamin, and zincobalamin. ${ }^{17)}$ These inhibitory effects were abolished by increasing concentrations of cyanocobalamin, suggesting that CFC and its derivatives are competitive inhibitors of cyanocobalamin. Conversion of CFC into the yellow product decreased the antimetabolite action of 
Table III. Cobalt-Free Corrinoid, Yellow Product, and Metal Analogues as Inhibitors of Cyanocobalamin-stimulated Growth of Vitamin B-12 Auxotrophic E. coli 215

\begin{tabular}{lc}
\hline Compound & Inhibition index $^{a}$ \\
\hline CFC $^{b}$ & 0.5 \\
Yellow product & 8.7 \\
Cu-analogue & 0.6 \\
Zn-analogue & No inhibition \\
\hline a & Ratio of concentrations of these compounds to \\
cyanocobalamin needed to $50 \%$ inhibition of the \\
growth of $E$. coli 215.
\end{tabular}

vitamin B-12. As it has been described that the corrin chromophore of the yellow product of hydrogenobyrinic acid a,c-diamide is interrupted between $\mathrm{C}-5$ and $\mathrm{C}-6$ through the formation of a lactam ring in ring $B,{ }^{17}$ the structural change of ring $\mathrm{B}$ may be responsible for the decrease of affinity for the cobalaminbinding protein. It is not understood why only the zinc analogue was inactive as an antimetabolite of cyanocobalamin in E. coli 215 .

\section{Effects of CFC on the growth of $M$. barkeri}

Stupperich et al. ${ }^{19)}$ reported that the copper analogue (cupribamide) did not stimulate the growth of $M$. thermoautotrophicum. Therefore, the effects of CFC-1 on the growth of $M$. barkeri were examined with or without cobalt chloride. As shown in Fig. 4, when CFC-1 was added to the basal medium at the concentration of $120 \mathrm{nM}$ with cobalt chloride $(2.5 \mu \mathrm{M})$, neither significant inhibitory nor stimulatory effects on the growth were observed. When $M$. barkeri was cultured on the cobalt-deficient medium after two subcultivations on the same medium, gas production decreased greatly. The slow gas production still observed might be due to the unavoidable cobalt contamination because the cells contained corrinoid at $266 \mathrm{nmol} / 1$ (data not shown). But, when CFC1 was added to the cobalt-deficient medium, gas production, hence growth, recovered to be similar to those in the cobalt-containing me-

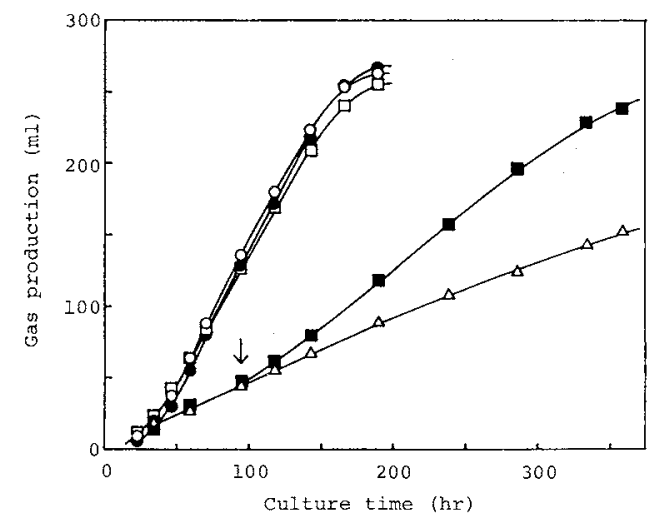

Fig. 4. Effect of Cobalt-free Corrinoid (CFC-1) on $M$. barkeri Fusaro with or without Cobalt Chloride.

Experimental vials (medium, $50 \mathrm{ml}$ ) were inoculated with $4 \%(\mathrm{vol} / \mathrm{vol})$ of a late lag phase culture grown twice on cobalt deficient medium (methanol, $8 \mathrm{~g} / \mathrm{l}$ ). Symbols: $\mathrm{O}$, basal medium $\left(\mathrm{CoCl}_{2}, 2.5 \mu \mathrm{M}\right) ; \triangle$, basal medium without $\mathrm{CoCl}_{2} ;$, at zero time, $\mathrm{CFC}$ was added at a concentration of $120 \mathrm{~nm}$ to the basal medium; $\square$, at zero time CFC was added at a concentration of $120 \mathrm{nM}$ to the basal medium without $\mathrm{CoCl}_{2} ; \boldsymbol{\square}$, at the time indicated by the arrow, CFC was added at a concentration of $120 \mathrm{~nm}$ to the basal medium without $\mathrm{CoCl}_{2}$.

dium in spite of the low concentration of CFC (120 nM).

Although the stimulatory effect of $\mathrm{CFC}$ on the growth of M. barkeri was not clear, it was speculated that the CFC was intracellularly converted to its metal analogues which could have coenzyme activity of a corrinoid in $M$. barkeri in a medium with a low level of cobalt but rich in other metals. This possibility was partially supported by the cobalamin activity of a Fe-analogue (ferribalamin) in Ochromonas malhamensis. ${ }^{18}$ In archaebacteria, a possible involvement of CFCs as intermediates in the biosynthesis of vitamin B-12, and coenzyme activity of CFC and metal analogues have not been sufficiently studied. In the future, the role of several metal analogues as coenzymes in methanogenesis may be confirmed by enzymatic studies.

Acknowledgment. We thank Dr. K. Sasaki for reading the manuscript and helpful discussion. 


\section{References}

1) J. I. Toohey, Proc. Natl. Acad. Sci. U.S.A., 54, 934 (1967).

2) J. I. Toohey, Fed. Proc., 25, 1628 (1966).

3) T. Kamikubo, K. Sasaki and M. Hayashi, J. Nutr. Sci. Vitaminol, 23, 179 (1977).

4) B. Dresow, G. Schlingmann, L. Ernst and V. B. Koppenhagen, J. Biol. Chem., 255, 7637 (1980).

5) K. Sato, S. Shimizu and S. Fukui, Biochem. Biophys. Res. Commun., 39, 170 (1970).

6) V. B. Koppenhagen and J. J. Pfiffner, J. Biol. Chem. Commun., 245, 5865 (1970).

7) V. B. Koppenhagen and J. J. Pfiffner, J. Biol. Chem. Commun., 246, 3075 (1971).

8) V. B. Koppenhagen, F. Wagner and J. J. Pfiffner, J. Biol. Chem., 248, 7999 (1973).

9) V. B. Koppenhagen, B. Elsenhans and F. Wagner, $J$. Biol. Chem., 249, 6532 (1974).

10) R. Carmel and V. B. Koppenhagen, Arch. Biochem.
Biophys., 184, 135 (1977).

11) J. Krzycki and J. G. Zeikus, Curr. Microbiol., 3, 243 (1980).

12) P. Scherer and H. Sahm, Acta Biotechnol, 1, 57 (1981).

13) T. K. Mazumder, N. Nishio, S. Fukuzaki and S. Nagai, Appl. Microbiol. Biotechnol., 26, 511 (1987).

14) T. K. Mazumder, N. Nishio, S. Fukuzaki and S. Nagai, Appl. Environ. Microbiol., 52, 617 (1986).

15) T. Kamikubo, Vitamin, 11, 43 (1956).

16) T. Kamikubo and H. Narahara, Vitamin, 38, 331 (1968).

17) V. B. Koppenhagen, in " $B_{12}$," Vol. 2, ed. by D. Dolphin, John Wiley \& Sons, Inc., New York, 1982.

18) R. Bieganowski and W. Friedrich, Proceedings of the Third European Symposium on Vitamin $\mathrm{B}_{12}$ and Intrinsic Factor, Zürich, Switzerland, ed. by $\mathbf{B}$. Zagalak and W. Friedrich, de Gruyter, Berlin, 1979, p. 647 .

19) E. Stupperich, I. Steiner and H. J. Eisinger, J. Bacteriol., 169, 3076 (1987). 